\title{
Introduciendo una historia interactiva en una experiencia geolocalizada
}

\section{Introducing an interactive story in a geolocalized experience}

\author{
José Rafael López Arcos ${ }^{1}$, Francisco Luis Gutiérrez Vela 2 . (iD), Natalia Padilla-Zea ${ }^{3}$, Patricia

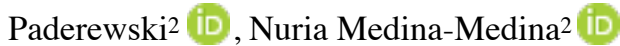 \\ ${ }^{1}$ Estudiante de Doctorado, Universidad de Granada, Granada, España \\ 2 Departamento de Lenguajes y Sistemas Informáticos, Universidad de Granada, España. \\ ${ }^{3}$ Instituto de Investigación, Innovación y Tecnología Educativas (iTED), Universidad Internacional de la Rioja, \\ España. \\ jrlarco@ugr.es, fgurierr@ugr.es, natalia.padilla@unir.net, patricia@ugr.es,nmedina@ugr.es
}

(Recibido 7 Diciembre 2017; aceptado 17 Enero 2018)

\begin{abstract}
Resumen. Actualmente, los juegos pervasivos son uno de los tipos de experiencia de juego que causan el mayor impacto en los jugadores, pues rompen el entorno usual del juego en su dimensión espacial, temporal o social. Este documento tiene el objetivo de proponer, en esos juegos, una integración de la narrativa con la interactividad y la geolocalización. Esta integración requiere un modelo que represente y estructure la historia y su fusión con el resto de elementos del juego. Además, ese modelo debe permitir analizar la efectividad de la historia y cómo ésta ayuda a crear una buena experiencia de usuario. En este trabajo, proponemos una fuerte estructuración de la narrativa que incluye soporte a la geolocalización. A través de un ejemplo de diseño de una historia interactiva geolocalizada, se explican las diferentes características del modelo que hacen posible la creación de buena narrativa interactiva.
\end{abstract}

Palabras clave: Narrativa, Juegos pervasivos, Narrativa geolocalizada, Experiencia de usuario, Videojuegos.

\begin{abstract}
Pervasive games are one of the types of gaming experiences that currently causes the most impact on players. They break the usual environment of the game in a spatial, temporal or social dimension. This paper has the objective of integrate the narrative with the interactivity and the geolocalization of these games. This integration requires a model that represents and structures the story and its joining between the rest of the elements of the game. In addition, this model should allow us to analyze the effectiveness of the story and how it helps to create a good user experience. In this paper, we propose a strong structuring of the narrative that includes geolocalization support. Through an example of the design of an interactive geolocalized story, we explain several characteristics of the model that make possible the creation of a good interactive narrative.
\end{abstract}

Keywords: Storytelling, Pervasive Games, Geolocalized Narrative, User Experience, Video Games.

\section{Introducción}

El juego ha sido siempre una herramienta útil para el aprendizaje, aunque también ha sido usado en muchos contextos diferentes, como rehabilitación, publicidad o marketing. Esto se debe, en mayor medida, a la gran motivación que despierta en el usuario/jugador. Actualmente, los juegos han sido adaptados a las nuevas tecnologías, consolidando el concepto de videojuegos o juegos digitales. La 
calidad de la experiencia de juego se ha incrementado significativamente debido al uso de nuevos dispositivos electrónicos que pueden ser usados para jugar (móviles, tabletas, relojes digitales, GPS, entre otros), que originan interés en todo tipo de usuarios, no sólo en jugadores habituales.

Los juegos pervasivos son uno de los tipos de experiencia de juego que, actualmente, causa mayor impacto en los jugadores. Estos juegos ofrecen mayor y mejor experiencia a los jugadores, al romper el entorno usual de juego [1]. Esto se conoce como ruptura espacial, temporal o social. La ruptura espacial se refiere a la extensión del espacio de juego fuera de los límites tradicionales, es decir, de la pantalla. Por otro lado, la ruptura temporal permite que los juegos no tengan definidos el momento de inicio o final, sino que el jugador puede jugar en cualquier momento e introducirse en las dinámicas de juego, sin tener que iniciar el juego en sí. De este modo, puede abandonarlo cuando quiera, sin frustración ni la sensación de no haberlo terminado. Por último, la ruptura social permite a los jugadores relacionarse con personas desconocidas, que son parte de la experiencia de juego. Incluso, pueden existir participantes del juego que no son conscientes de estar jugando, pero cuyas acciones afectan al desarrollo del juego. En los juegos pervasivos, es usual mezclar realidad con ficción, expandiendo el mundo del juego mediante elementos como objetos o lugares de la realidad, la posición de los jugadores o acciones y situaciones que ocurren fuera del juego.

La mayor parte del potencial de los juegos pervasivos radica en que se benefician de procesos y elementos que normalmente no vemos en los juegos; por ejemplo, ejercicio físico, relaciones sociales, creación colaborativa de historias, entre otros.

De todos los juegos pervasivos, podemos centrarnos en los llamados juegos basados en localización o juegos geolocalizados. Este tipo de juegos expanden la dimensión espacial, es decir, la experiencia incluye elementos del juego distribuidos por lugares y situaciones reales. El jugador utiliza un dispositivo para comunicarse con el mundo del juego, pero este se desarrolla tanto en escenarios virtuales como reales. Este tipo de experiencia, que llamamos experiencia geolocalizada, tiene numerosos campos de aplicación en educación, marketing, turismo, etcétera [2].

A partir de la experiencia proporcionada por diseñadores de videojuegos [3], sabemos que, para que un juego atraiga al jugador durante un tiempo razonable y los resultados deseados sean obtenidos, es necesario que el juego presente una narrativa que permita que la experiencia sea satisfactoria. Es decir, que el usuario se integre de forma natural en el juego. Además, ya que, probablemente, no todos los usuarios tienen la misma experiencia, la narrativa actúa como un elemento integrador, de forma que pueda adaptarse a cada usuario en tiempo real. Por ejemplo, diferentes partes de la historia pueden ser presentadas dependiendo de las acciones, decisiones o preferencias del jugador.

Para ello, es necesario estructurar apropiadamente la narrativa definida para el juego y conseguir fragmentos de historia de diferentes niveles de motivación. Esta medida es dada por el análisis de los componentes de la historia y la instanciación de esos componentes en una experiencia particular, con el objetivo de ofrecer a los jugadores una buena narrativa. De este modo, los diferentes componentes de la narrativa pueden ser combinados de diferentes maneras, con una lógica apropiada, evitando historias rígidas y estáticas.

Finalmente, es necesario evaluar la experiencia de los jugadores en este tipo de juegos, de modo que se obtenga información sobre la efectividad de la narrativa implementada. Esto permite adaptar o cambiar la narrativa, tanto en fase de diseño, como en tiempo de juego.

El presente trabajo se estructura de la siguiente manera. Las secciones segunda y tercera brindan, respectivamente, información sobre el análisis de la experiencia de usuario y la narrativa interactiva en experiencias geolocalizadas. La cuarta sección presenta nuestra proposición de modelado de la narrativa, especialmente varias características que ayudan a diseñar una buena narrativa interactiva geolocalizada. Además, se describe cómo implementar gráficamente los diagramas acordes a dicha estructuración, en el diseño de una experiencia geolocalizada. La sección quinta muestra un ejemplo de uso del modelo y cómo se implementan las características de este. La última sección muestra las conclusiones y el trabajo futuro. 


\section{Análisis de la experiencia de usuario en experiencias geolocalizadas}

Al desarrollar un videojuego, un aspecto fundamental es tener en cuenta a los jugadores potenciales, es decir cuáles son sus gustos y motivaciones y cómo encajan estas con la experiencia que se quiere proporcionar durante el juego. Uno de los retos más importantes para los diseñadores es analizar y comprender qué hace divertido un juego y cómo se genera una buena experiencia en el jugador. En el área de la interacción persona-ordenador, la experiencia de usuario ha sido estudiada extensamente en el uso de sistemas interactivos y se han propuesto diferentes formas de medirla y analizarla. Esta idea se aplica al caso de los juegos, donde se usa el término experiencia del jugador y se proponen medidas similares, como diversión, motivación o jugabilidad [4].

En el caso de los videojuegos que tienen gran dependencia de la narrativa, tales como juegos de rol o aventuras gráficas, podemos observar cómo la eficiencia de la narrativa tiene una importancia relevante en el análisis de la experiencia de juego, tanto a nivel de diversión como de atracción e implicación (o engagement) del jugador. En gran parte, esta experiencia es dada por la inmersión que provoca la historia en los jugadores, haciéndoles participar en las situaciones que viven los personajes. Sin olvidar que el elemento diferenciador del videojuego es la interactividad y, por lo tanto, que el jugador debe tener una capacidad de decisión importante en el desarrollo de la narrativa.

Actualmente, nuevas visiones de juego están siendo exploradas, donde las historias están parcialmente pre-diseñadas y preparadas para emerger dinámicamente (narrativas emergentes) [5]. En este sentido, las decisiones del jugador marcan la trama y tienen una fuerte influencia en la intensidad narrativa percibida.

Desde el punto de vista teórico, la integración de la historia con la interactividad del juego requiere una acción a dos niveles. Por un lado, es necesario un modelo que represente y estructure la historia y su integración con el resto de elementos del juego. Por otro, este modelo debe permitirnos analizar la efectividad de la historia y cómo esta ayuda a crear la experiencia que el jugador vivirá.

En experiencias pervasivas, es necesario analizar las características tanto del tipo de juego como de la tecnología usada. Así, podemos entender por qué los jugadores obtienen diversión y motivación y cómo les afectan estas nuevas características. El contexto tiene un papel importante en este tipo de juegos y hace mucho más complejo el diseño de los entornos donde el jugador vivirá su aventura.

\section{Narrativa interactiva y geolocalizada}

Como se ha mencionado, es fundamental que, en los videojuegos, particularmente los pervasivos, la narrativa se convierta en una parte importante de la experiencia del jugador. Esta narrativa debe ser integrada con los otros elementos del juego, especialmente con las mecánicas que permiten al jugador interactuar con el mundo del juego.

La narrativa digital interactiva lleva al jugador más cerca de la acción, permitiendo que sus decisiones afecten el transcurso de la historia. Esta libertad otorgada al jugador provoca una aparente pérdida de control sobre la historia por parte del escritor. Esta pérdida de control es aún mayor en el caso de las experiencias geolocalizadas. Adicionalmente, como se establece en [6], una experiencia geolocalizada debe suavizar la diferencia entre realidad y ficción integrando ambas en una realidad alternativa.

El entorno definido por las reglas y elementos del videojuego se llama espacio de juego. En el caso de los juegos geolocalizados, los espacios virtual y real se mezclan de forma que las acciones en uno pueden tener consecuencias en el otro. Cuando la narrativa del juego tiene gran peso en el diseño, el espacio de juego se convierte en un espacio narrativo y es controlado por el jugador, para que este perciba, al menos, que posee dicho control. 
El espacio físico da al jugador un contexto donde elementos como la historia, la cultura o las relaciones sociales generan nuevas oportunidades y restricciones. Estas influyen en sus posibles acciones e interacciones, tanto en el juego como en la evolución narrativa.

En la literatura, podemos encontrar varias soluciones a la incorporación de la narrativa en experiencias geolocalizadas. En [7], los autores usan una narrativa lineal que el jugador lee y escucha a lo largo de la experiencia. Para controlar la manera en que el jugador recibe dicha historia, la herramienta lleva un control del porcentaje de historia que el jugador ha leído. En realidad, esos contenidos no son una narrativa pura, sino un conjunto de contenidos educativos que los autores presentan como vida, historia y arquitectura.

Los autores de [8] dividen la narrativa en pequeñas mini-historias, cada una asignada a un lugar en la experiencia. En este caso, el texto se divide mediante enlaces para viajar por el espacio de la experiencia. El itinerario seguido por un viajante correspondería, entonces, con una instancia de la historia. En esta propuesta, localización y tiempo son considerados claves esenciales para trabajar. Se menciona que la experiencia ocurre a lo largo de diferentes espacios geolocalizados, donde el jugador tiene que ir. El objetivo de este diseño es hacer que la experiencia se convierta en una concepción semántica del lugar, ya que, según proponen, cuando un espacio es percibido como familiar, se convierte en un lugar. Esto significa que recibir información sobre un espacio real permite a las personas comprenderlo mejor y convertirlo en un lugar memorable.

En esta experiencia, los usuarios disfrutan de un estilo de historia autocontenido y de poder ser capaces de elegir qué experimentarán seguidamente. Sin embargo, los fragmentos pequeños pueden reducir la profundidad narrativa: un conjunto de historias no es una narrativa completa. Este resultado refuerza la idea de la necesidad de dar cohesión a la historia interactiva a lo largo de todo el conjunto de eventos narrativos, que deben ser analizados y controlados.

Por otro lado, en el trabajo propuesto en [9], el arco narrativo puede ser mapeado en un arco preexistente, enlazado con la distribución espacial del lugar. En este caso, se crea una experiencia que lleva la atención al contexto invisible de la historia y hace que el jugador se vea inmerso en el descubrimiento.

Respecto a la creación de una arquitectura para soportar la experiencia geolocalizada con narrativa, el trabajo de [10] establece que se debe: 1) ser capaz de dar soporte a la narrativa con una localización específica; 2) tener la habilidad de gestionar historias ramificadas y no lineales; y 3) proporcionar una gestión eficiente de recursos.

En dicho trabajo, la narrativa está basada en el concepto de reto como parte atómica de la historia, por ejemplo, una tarea que el jugador ha de completar. Cada uno de esos retos tiene una precondición, una condición de fallo y una condición de éxito. Un reto se "activa" cuando su precondición es alcanzada.

Sin embargo, como presentamos en la siguiente sección, en el presente trabajo entendemos la narrativa como una capa separada, diferente de los retos de juego. La narrativa es capaz de unificar el videojuego más allá de su conjunto de retos. Adicionalmente, pensamos que la libertad dada al jugador es importante, lo que significa que, tanto el diseño y estructura de la historia, como la efectividad generada en los jugadores, se vuelven más complejos.

Proponemos una estructuración jerárquica basada en grafos, con el objetivo de facilitar, en primer lugar, la creación de contenidos narrativos para experiencias geolocalizadas y, luego, el análisis de dichas experiencias. Esta estructura está basada en un modelo conceptual de la narrativa, presentado en la siguiente sección.

\section{Propuesta de estructuración y representación de la narrativa geolocalizada}

En la presente sección, se explica nuestra propuesta de diseño de la narrativa interactiva geolocalizada, a través de una fuerte estructuración. Además, se explican las características otorgadas a la narrativa 
gracias a esta estructuración. En último lugar, se especifica un método de representación gráfica de narrativas interactivas para el diseño de experiencias geolocalizadas.

\subsection{Dando estructura a la narrativa interactiva geolocalizada}

Como se ha mencionado, la narrativa interactiva presenta el problema de la pérdida de control por parte del autor sobre el curso de la historia, que es decidido por el espectador. Además, en las experiencias geolocalizadas, la libertad de movimiento en el mundo real es esencial para fortalecer la inmersión de los jugadores en el juego. En consecuencia, es especialmente necesario crear una historia que mantenga equilibrio entre la libertad del jugador y el control del autor, para que pueda ofrecer una historia de calidad. Con el objetivo de facilitar el diseño de este tipo de narrativa interactiva, proponemos el modelo conceptual de la Figura 1.

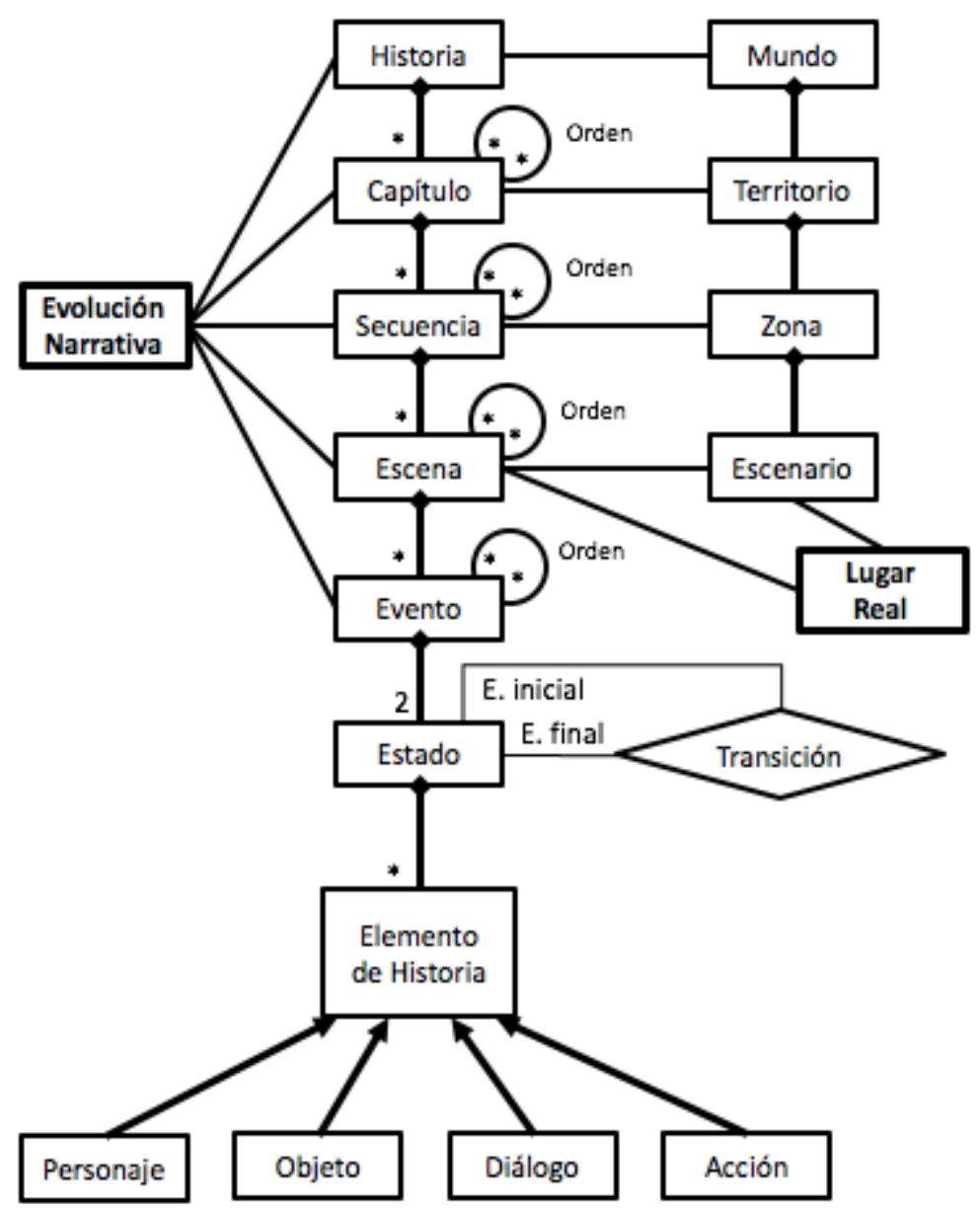

Figura 1. Modelo Conceptual de la narrativa interactiva geolocalizada.

Este modelo comienza por una narrativa fragmentada no lineal. Estructura fuertemente la historia y la manera en que el juego puede generar una evolución narrativa (casi siempre guiada por el jugador). En este sentido, un conjunto de estructuras de orden da significado a la historia y pueden ser controladas durante el iuego para obtener experiencias más efectivas. De hecho. el modelo define el espacio narrativo 
del juego, que debe ser considerado en conjunto con la estructura virtual de juego y su relación con las localizaciones físicas para componer el espacio de juego completo.

A continuación, se describen brevemente los elementos presentes en el modelo. Estos elementos están definidos en un diccionario general de historia. La Tabla 1 muestra el modelo de escena de dicho diccionario, como ejemplificación de la definición de los siguientes elementos:

Historia, capítulo, secuencia, escena: son fragmentos de historia ordenados jerárquicamente. Existe una relación de orden entre los fragmentos de la misma jerarquía.

Mundo, región, zona, escenario: son los lugares donde ocurren, respectivamente, la historia, los capítulos, las secuencias y las escenas.

Lugar real: en historias geolocalizadas, un escenario de la historia puede estar ligado a un lugar en el mundo real. Del mismo modo, existen escenas con carga narrativa que ocurren solo en la realidad.

Evolución narrativa: hechos concretos que ocurren en la historia.

Evento: un conjunto de eventos forma una escena. Los eventos se relacionan entre sí mediante relaciones de orden. Un evento es una transición entre dos estados de la historia. Ese cambio en el estado de la historia provoca un avance narrativo. El estado inicial y final de un evento puede ser usado como pre-condiciones y post-condiciones para avanzar sobre la historia no-lineal.

El estado de la historia: consiste en un conjunto de elementos de historia (personajes, objetos, diálogos y acciones) y sus respectivos estados actuales.

Tabla 1. Modelo de Escena.

\begin{tabular}{|c|c|c|}
\hline Atributo & Descripción & Dominio \\
\hline Identificador & Identificador interno. & $\mathrm{x}: \mathrm{x} \in[\mathrm{SC} 00000, \mathrm{SC} 99999]$ \\
\hline $\begin{array}{l}\text { Nombre } \\
\text { general }\end{array}$ & $\begin{array}{l}\text { Nombre general que describe la escena y funciona como clave } \\
\text { de diccionario. }\end{array}$ & $\mathrm{x}: \mathrm{x}$ es Nombre General \\
\hline $\begin{array}{l}\text { Evolución } \\
\text { narrativa }\end{array}$ & Descripción de lo que ocurre en la escena & Lenguaje natural \\
\hline Eventos & Conjunto de eventos que componen la escena & $\{\mathrm{x}\}: \mathrm{x}$ es Evento \\
\hline Escenario & Escenario donde la escena tiene lugar & $\mathrm{x}: \mathrm{x}$ es Escenario \\
\hline Lugar real & Lugar del mundo real donde puede ocurrir la escena & $\mathrm{x}: \mathrm{x}$ es Lugar Real \\
\hline $\begin{array}{l}\text { Escenas } \\
\text { previas }\end{array}$ & Conjunto de escenas que deben ocurrir antes de esta escena & $\{x\}: x$ es Escena \\
\hline $\begin{array}{l}\text { Secuencias } \\
\text { previas }\end{array}$ & Conjunto de secuencias que deben ocurrir antes de esta escena & $\{\mathrm{x}\}: \mathrm{x}$ es Secuencia \\
\hline $\begin{array}{l}\text { Capítulos } \\
\text { previos }\end{array}$ & Conjunto de capítulos que deben ocurrir antes de esta escena & $\{\mathrm{x}\}: \mathrm{x}$ es Capítulo \\
\hline Obligatoria & $\begin{array}{l}\text { Indica si la escena debe ocurrir obligatoriamente para que la } \\
\text { historia tenga sentido }\end{array}$ & $\mathrm{x}: \mathrm{x} \in\{\mathrm{Sí}, \mathrm{No}\}$ \\
\hline $\begin{array}{l}\text { Escenas } \\
\text { gemelas }\end{array}$ & $\begin{array}{l}\text { Conjunto de escenas con la misma función narrativa que esta } \\
\text { escena, pero que son diferentes y pueden sustituirla, debido a } \\
\text { diferentes razones (evitar repetición, coherencia con el resto de } \\
\text { la instancia de historia, dar mayor o menor información al } \\
\text { jugador, etc.) }\end{array}$ & $\{x\}: x$ es Escena \\
\hline
\end{tabular}


Las secciones 4.1.1 a 4.1.6 describen las características principales de las historias geolocalizadas, creadas según el modelo propuesto: no linealidad, jerarquía, control de la narrativa interactiva, evaluación de la narrativa, localización de la narrativa en el mundo real y soporte de experiencias reales.

\subsubsection{No linealidad}

Una historia no lineal está compuesta de partes interconectadas mediante relaciones de orden. Esta estructuración define un grafo cuya navegación lleva a diversas historias. De este modo, diferentes jugadores generan diferentes historias. A cada una de estas, generada durante una experiencia de juego, la llamamos instancia de historia.

\subsubsection{Jerarquía}

Si una historia está compuesta de capítulos interconectados, dentro de uno de estos encontramos también una estructura de secuencias con forma de grafo dirigido. Del mismo modo, estas secuencias se componen cada una de un grafo de escenas. Una vez más, un grafo de eventos describe cada escena en términos de los hechos que ocurren durante la escena. El evento es, finalmente, la parte más simple de la estructura narrativa, definido como una transición entre dos estados de la historia. El estado de la historia es el conjunto de los estados (cómo están) de cada elemento narrativo (personajes, objetos, escenarios y diálogos).

Esta estructuración jerárquica permite a los diseñadores trabajar con diferentes niveles de abstracción. De este modo, en historias grandes o complejas, se evita trabajar con grandes grafos. poco manejables. A la vez, ayuda a estructurar conceptualmente la historia.

\subsubsection{Control de la narrativa interactiva}

Debido a la naturaleza jerárquica del modelo, una escena empieza y termina con uno o varios eventos. Al consistir los eventos en un estado inicial y otro final, dichos estados actúan como condiciones del evento. Por tanto, el inicio de la escena tiene asociados una o varias pre-condiciones y una o varias postcondiciones. Lo mismo se aplica en el caso de las secuencias o capítulos. Estas condiciones son útiles para saber si una parte de la historia debe, o no, ocurrir en un momento particular del tiempo de juego. Además, pueden ser usados para modificar la historia en tiempo real.

Para ilustrar estos conceptos, imaginemos un ejemplo en el que el jugador entra en una habitación. En este caso, el estado inicial, definido como el conjunto de los elementos de la historia y su estado actual, podría ser: luz encendida, armario cerrado y diario oculto. Si el jugador abre el armario, esta transición hace que cambie el estado de la historia: el armario está abierto y el diario aparece dentro. Este cambio implica una evolución narrativa. Es un evento.

De forma similar, otras acciones, como coger el diario, leerlo, etc., producen diferentes eventos que hacen avanzar la historia. Pero, si el jugador no encuentra el diario y continúa explorando otros escenarios (que pueden llevarlo, incluso, a otros capítulos de la historia), puede ocurrir que las precondiciones de alguna escena no se cumplan, en algún momento, porque el jugador debería conocer los datos escritos en el diario. En este caso, el autor puede implementar una de las siguientes soluciones, dependiendo del caso específico: (a) la historia continúa, con las consecuencias de no haber leído el diario; (b) el jugador debe dejar esa rama de la historia y volver a la habitación donde estaba el diario, para poder retomar esa rama más tarde; o (c) se usa algún recurso narrativo (que se puede implementar mediante una escena gemela), para dar al jugador la información necesaria. Todas estas soluciones son fácilmente controlables, gracias a la estructura jerárquica y al uso de pre y post-condiciones, incluidas en el modelo.

Por tanto, las pre-condiciones son características que el estado de la historia debe cumplir para poder acceder a ese evento. Las post-condiciones son, por su lado, modificaciones que ha provocado el evento en el estado de la historia. 


\subsubsection{Evaluación de la narrativa}

El modelo propuesto convierte la narrativa interactiva en una estructura jerárquica, formada por grafos. De este modo, los nodos del grafo representan partes de la historia, a cada uno de los cuales es posible asignar datos sobre su importancia, función narrativa o intensidad emocional, entre otros. De este modo, dichos datos pueden ser usados para realizar una evaluación, que puede ser realizada durante el proceso de creación de la historia.

Debido a que es fácil obtener los diferentes caminos entre dos nodos de un grafo, las diferentes instancias de historia que pueden surgir de la estructura narrativa pueden ser pre-evaluadas, para poder rediseñar las partes débiles. Además, la evaluación puede ser realizada durante el tiempo de juego. En este caso, el resultado de la evaluación puede emplearse como precondición para limitar, o dar luz verde, a ciertos eventos. Ambos modos deben ser diseñados cuidadosamente, para mejorar la calidad de la historia que cada jugador recibe [11].

\subsubsection{Localización de la narrativa en el mundo real}

En el modelo propuesto, la estructuración de la narrativa debe corresponder con la estructuración del mundo imaginario, en el que ocurre la historia, ya que una escena ocurre en un escenario; una secuencia (o conjunto de escenas), en una zona; un capítulo, en un territorio; y la historia, en el mundo. Cuando estos lugares virtuales tienen correlación con el mundo real, se obtiene una historia geolocalizada. El caso más claro es la realidad virtual, donde están completamente unidos el escenario real e imaginario. Sin embargo, esta integración puede no ser tan explícita.

Tal posible relación entre la historia virtual y el mundo real está soportada por el modelo propuesto. Para ello, en el modelo de la Figura 1, se puede observar la relación existente entre el escenario de la historia y el lugar real. De este modo, la escena que ocurre en ese escenario virtual tiene una relación explícita con el mundo real.

Además, esta integración de la historia con la realidad se manifiesta en el uso de pre-condiciones o post-condiciones, basadas en elementos del mundo real. Por ejemplo, una pre-condición puede basarse en que el jugador haya visitado un lugar, o realizado una acción sobre un objeto real. En ese caso, se entendería que, al ejecutar la acción, se le ha narrado parte de la historia. Si el jugador no conoce esa parte de la historia, se le deberá narrar de una forma alternativa. Llamamos escena gemela a aquellas que aparecen en el diagrama de forma paralela y cuya utilidad es narrar la misma parte de la historia, de formas diferentes, dependiendo de los actos previos del jugador. Así, conseguimos que se pueda narrar lo mismo en diferentes lugares reales, para otorgar más libertad al jugador.

\subsubsection{Soporte de experiencias reales}

En una experiencia geolocalizada, el usuario vive experiencias reales en lugares físicos integrados con una historia fantástica. De hecho, los eventos que ocurren en el lugar real pueden generar evolución narrativa en la historia. Como se observa en la Figura 1, los eventos generan evolución narrativa y hacen que, cuando la experiencia avanza, el jugador reciba más información sobre la historia. Un evento puede ser parte de una escena relacionada con un lugar real y corresponder a una actividad física realizada por el jugador.

En muchas situaciones, esta evolución es imposible de pre-diseñar y las actividades derivadas no pueden ser directamente controladas por el juego. Sin embargo, esas interacciones enriquecen enormemente la experiencia del jugador. En cualquier caso, la estructura narrativa debe tener en cuenta este aspecto crucial, derivado de la interacción con la realidad. Por tanto, una escena representada en nuestro diagrama de escenas puede ser puramente real, al igual que sus eventos, es decir, no existe un control explícito por parte del videojuego. Esta característica se observa en la relación existente entre escena y lugar real en el diagrama de la Figura 1.

La estructuración narrativa soporta el añadido de información sobre la intensidad emocional de cada parte de la historia. Esta anotación semántica puede ser usada para evaluar la evolución narrativa real en 
comparación con la esperada y, si es necesario, introducir nuevos personajes o situaciones que provoquen intensidad narrativa (por ejemplo, mayor intriga). Estos cambios en tiempo de juego pueden servir para mantener la atención del jugador, mejorando la experiencia generada por el juego.

\subsection{Representación gráfica de la historia}

Como se ha mencionado, para definir una estructura narrativa, construimos un grafo. En este, los elementos de la estructura narrativa (eventos, escenas, secuencias o capítulos) se representan mediante nodos. Las relaciones de orden se representan mediante flechas, que unen dichos nodos. Observando el modelo conceptual de la Figura 1, se aprecia que solo dos elementos del mismo tipo pueden tener una relación de orden entre sí (un evento solo puede estar relacionado con otro evento, una escena con otra escena, etc.). Por tanto, un nodo A, relacionado con un nodo B mediante una relación de orden, implica que la evolución narrativa asignada a A debe anteceder en la historia a la evolución narrativa asignada a B. La Tabla 2 muestra cómo representar los nodos y las relaciones, cuando se estructura una historia interactiva geolocalizada.

Hemos expuesto el carácter jerárquico del modelo y el concepto de estado. Los estados iniciales y finales permiten llevar a cabo el control de la narrativa interactiva, como se ha mencionado. Con estos conceptos se puede comprender la creación de una estructura narrativa. La Figura 2 muestra un ejemplo de la estructura narrativa de una posible escena, que consiste en un conjunto de eventos, relacionados entre sí, mediante relaciones de orden. En dicha figura, se observa que:

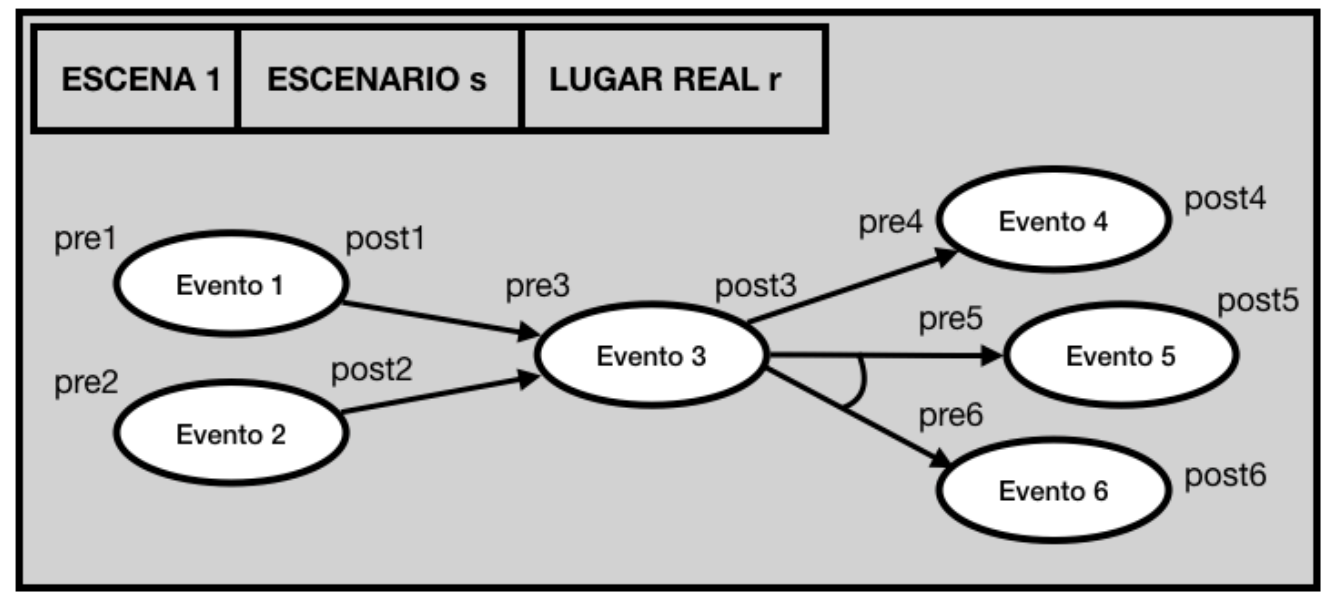

Figura 2. Ejemplo de estructura narrativa de una escena.

- Los eventos 1 y 2 no tienen relación de orden entre sí, por lo que pueden realizarse, indistintamente, en cualquier orden. Es decir, el estado final del evento 1 no va a influir en el estado inicial de evento 2 y viceversa.

- La suma de los estados finales (las post-condiciones) del evento 1 y el 2 está incluida en el estado inicial (la pre-condición) del evento 3.

- La post-condición del evento 3 está incluida en las pre-condiciones de los eventos 4, 5 y 6 .

- El evento 4 es, según este diagrama, un camino de la historia excluyente del camino formado por los eventos 5 y 6 . Si no se cumplen las pre-condiciones del evento 4 , se narrarán los eventos 5 y 6 , o viceversa.

- Los eventos 5 y 6 podrán realizarse indistintamente, en cualquier orden, pero ambos una vez se ha narrado el evento 3 . 
Tabla 2. Diagramas de historia: representación gráfica.

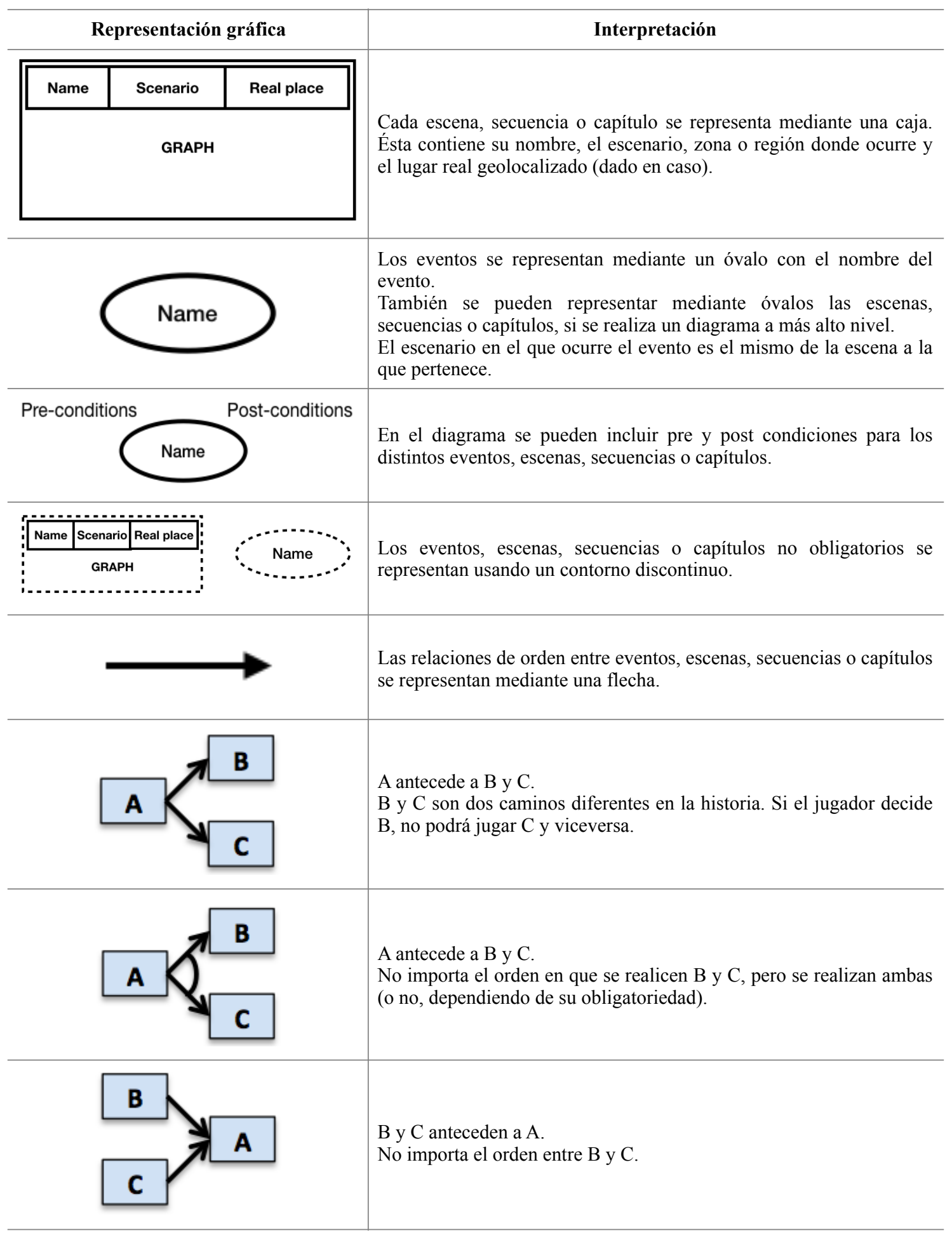


- Al principio de la escena, existe un estado de la historia que, asumimos, incluye las precondiciones de los eventos 1 y 2 y un conjunto de posibles estados diferentes de la historia al final de la escena. Esto se debe a la modificación que sufre el estado por los sucesivos eventos, ya que las post-condiciones de cada evento se suman al estado de la historia. En primer lugar, a que los eventos 4 , por un lado, y 5 y 6 , por otro, son excluyentes. Además, es posible que el orden en que se realizan dos eventos (en este caso, los eventos 1 y 2 , o los eventos 5 y 6 ) influya en el resultado de la suma de los estados finales de dichos eventos.

Cabe explicar que existe la posibilidad de que, habiendo realizado los eventos que en el diagrama preceden a un evento particular, no se cumpla aún la pre-condición de dicho evento. Esto se puede deber a que dicha pre-condición también supone haber cumplido con partes de la historia que están en otro lugar del grafo. Este uso de las precondiciones crea una red de relaciones indirectas, que permite llevar a cabo un control de la narrativa interactiva aún más extenso y complejo. En el ejemplo de la sección 5, se muestran casos concretos de esta característica.

Del mismo modo que hemos construido una escena mediante eventos, se puede construir una secuencia mediante escenas o un capitulo mediante secuencias. Cada una tendrá un estado inicial y un conjunto de posibles estados finales de la historia. La jerarquía propia del modelo puede ser representada gráficamente en estos diagramas.

\section{Diseño de una experiencia interactiva y geolocalizada}

Este diseño se basa en el juego presentado en [12], el cual consiste en una experiencia geolocalizada soportada por dispositivo móvil. El objetivo de dicha experiencia es guiar al jugador a lo largo de una visita a varios edificios de la Universidad de Granada. Concretamente, el jugador tiene que visitar los siguientes edificios: las facultades de derecho, filosofía, ciencias del deporte, farmacia, arquitectura e ingeniería informática. La historia que da soporte a la experiencia cuenta cómo un científico loco quiere dar vida a un monstruo, al que está construyendo mediante piezas proporcionadas por diferentes personajes en diferentes lugares (ver Figuras 3 y 4 ).

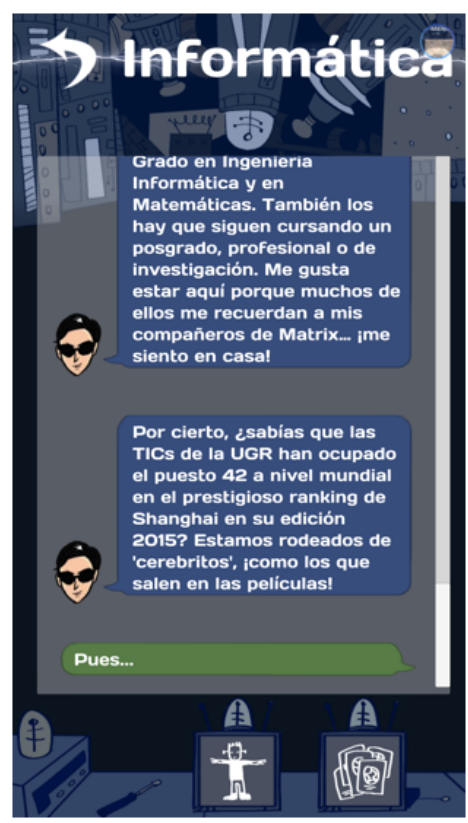

Figura 3. Conversación con un personaje en el videojuego LivingUGR. 

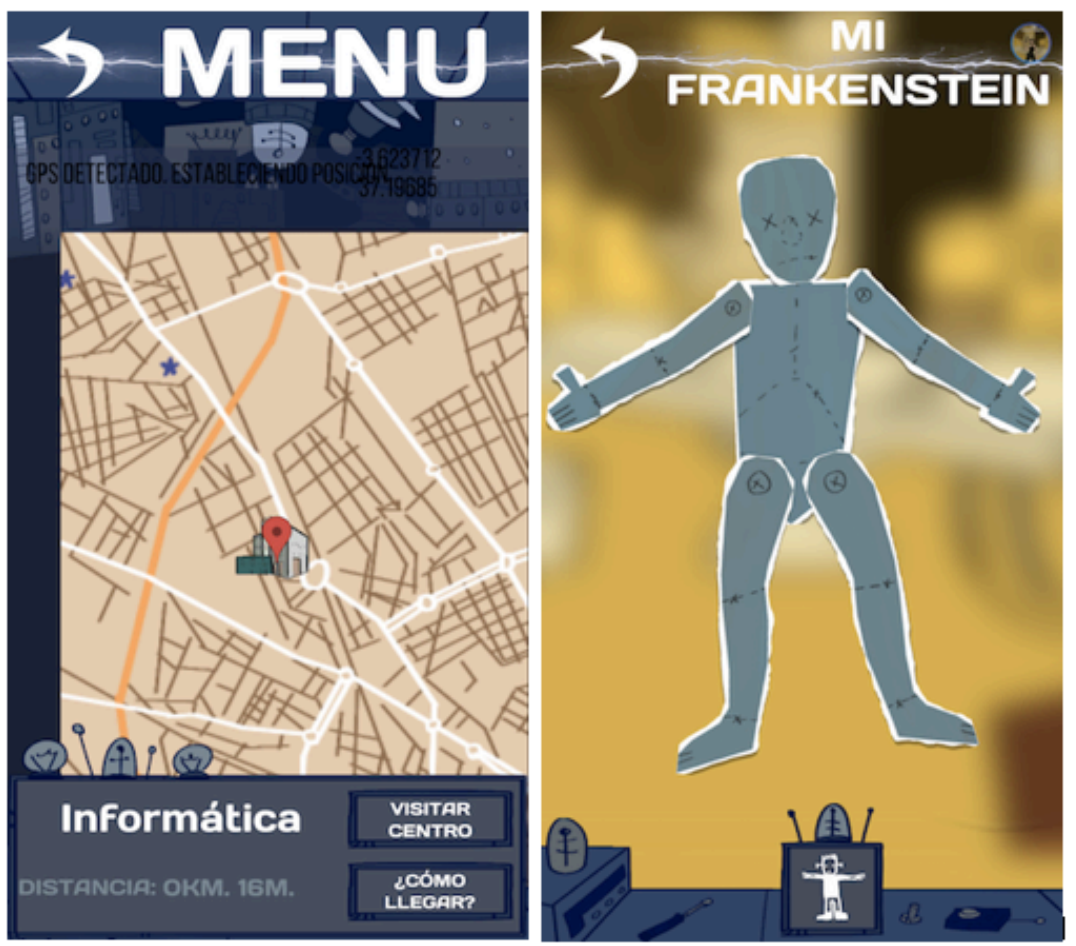

Figura 4. Construyendo el monstruo y encontrando localizaciones en el mundo real en el videojuego LivingUGR.

Sin embargo, nos dimos cuenta de que es interesante enlazar de forma más explícita la historia con elementos en el mundo real, así como ofrecer una historia más compleja de acuerdo al público objetivo (estudiantes pre-universitarios). Así, en este trabajo, presentamos una parte del diseño de esta nueva historia: una competición de monstruos se va a celebrar y varios científicos locos construirán sus propios monstruos. Para ello, necesitan mejorar su conocimiento y la manera más adecuada para ello consiste en visitar las diferentes facultades de la Universidad de Granada.

En esta sección, como ejemplo, nos centramos en el proceso de construir uno de esos monstruos y la Figura 5 muestra que la información necesaria para hacerlo está distribuida en varias facultades (algunas de las secuencias están etiquetadas con nombres de lugares reales):

Nuestro científico loco necesita toda esa información, pero no puede visitar todos los lugares. Así, el juego adaptará la historia a los lugares que el jugador visite. En este capítulo de la historia, se narra al jugador que necesita obtener piezas de cuerpo de monstruo para construirlo. Para conseguirlas, debe ir a la Facultad de Artes, donde encontrará que muchas esculturas con imperfecciones se guardan en un almacén y pueden ser usadas como partes del cuerpo. No parecerá un ser humano, pero puede obtener una solución química para ese problema (Facultad de Ingeniería química). Una vez tiene las piezas, necesita darles vida y una buena opción es ir al hospital. Cuando llega, escucha a ciertos médicos hablando de una operación compleja, que necesita del desfibrilador para reanimar al paciente. Cuando lo encuentra, un técnico le dice que la máquina puede revivir, pero necesita mucha más potencia (cerca de 1 gigavatio) para dar vida a un monstruo. Así que debe ir a la facultad de Ingenierías Informáticas y de Telecomunicaciones. Allí, visita el edificio y los laboratorios. En uno de ellos, unos investigadores están trabajando en un proyecto en el que usan una antena para capturar rayos de tormenta, los cuales tienen 1.21 gigavatios, ¡suficiente para dar vida al monstruo! La solución al problema es construir una antena como esa... o robarla. Si se desea construir, se necesita una serie de materiales de la Facultad de Ciencias, del Grado en Electrónica; si se quiere robar, el jugador debe aprender técnicas para capturar ladrones y así poder evitar ir a la cárcel (Facultad de Criminología y Facultad de Derecho). Una vez consiga la antena, el monstruo viviente podrá ser una realidad. 


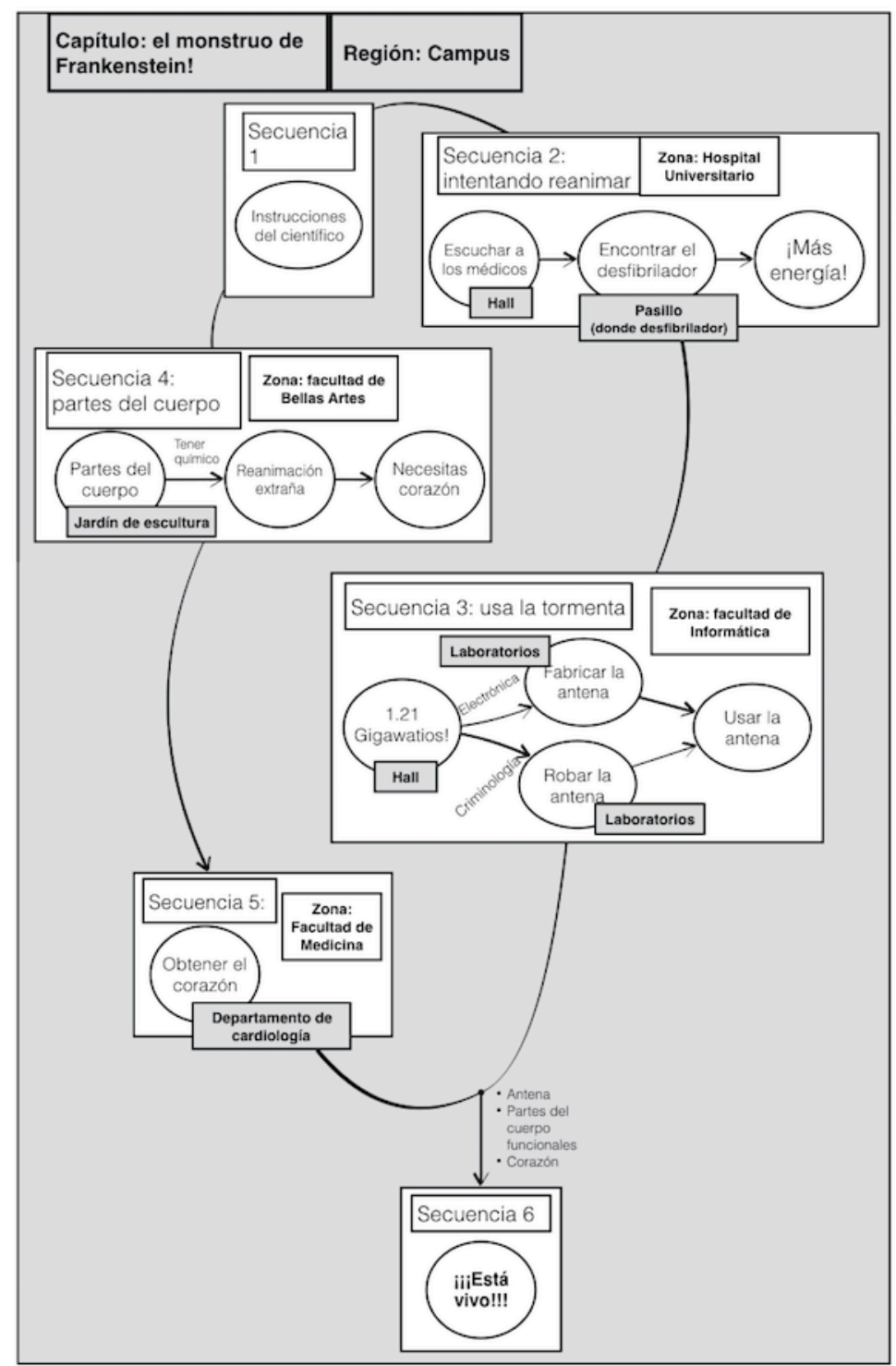

Figura 5. Ejemplo de estructuración de un capítulo de una historia interactiva geolocalizada.

A continuación, se describe cómo este ejemplo presenta las características del modelo previamente descrito:

No linealidad: el jugador puede elegir el orden en el que quiere visitar los lugares reales. De hecho, recibirá la historia en el orden en el cual los lugares son visitados.

Jerarquía: en la Figura 5, se puede observar la estructura de secuencias (rectángulos blancos) que forman este capítulo de la historia. Cada secuencia tiene una estructura de escenas, representadas mediante elipses, pues son el elemento más simple de este diagrama, plasmado a nivel de capítulo (por simplificar). Cada escena está localizada en escenarios, que pueden estar ligados o no a lugares reales.

En la Figura 6, se muestra una vista a nivel de secuencia de la secuencia 3. En ella se pueden observar con detalle las escenas que la forman, sus escenarios y lugares reales y el grafo de eventos que compone cada una.

Control de la narrativa interactiva: tras el comienzo del capítulo (secuencia 1), el jugador puede visitar las facultades en cualquier orden. Además, hay capítulos que pueden ser jugados antes y después de este (hay capítulos donde visitamos el Departamento de Electrónica, el de Criminología, o la Facultad de Química, que pueden influir en este capítulo). Las pre y post condiciones de cada evento y escena permiten controlar este hecho. Estas condiciones están etiquetadas en algunas transiciones entre escenas o secuencias. 


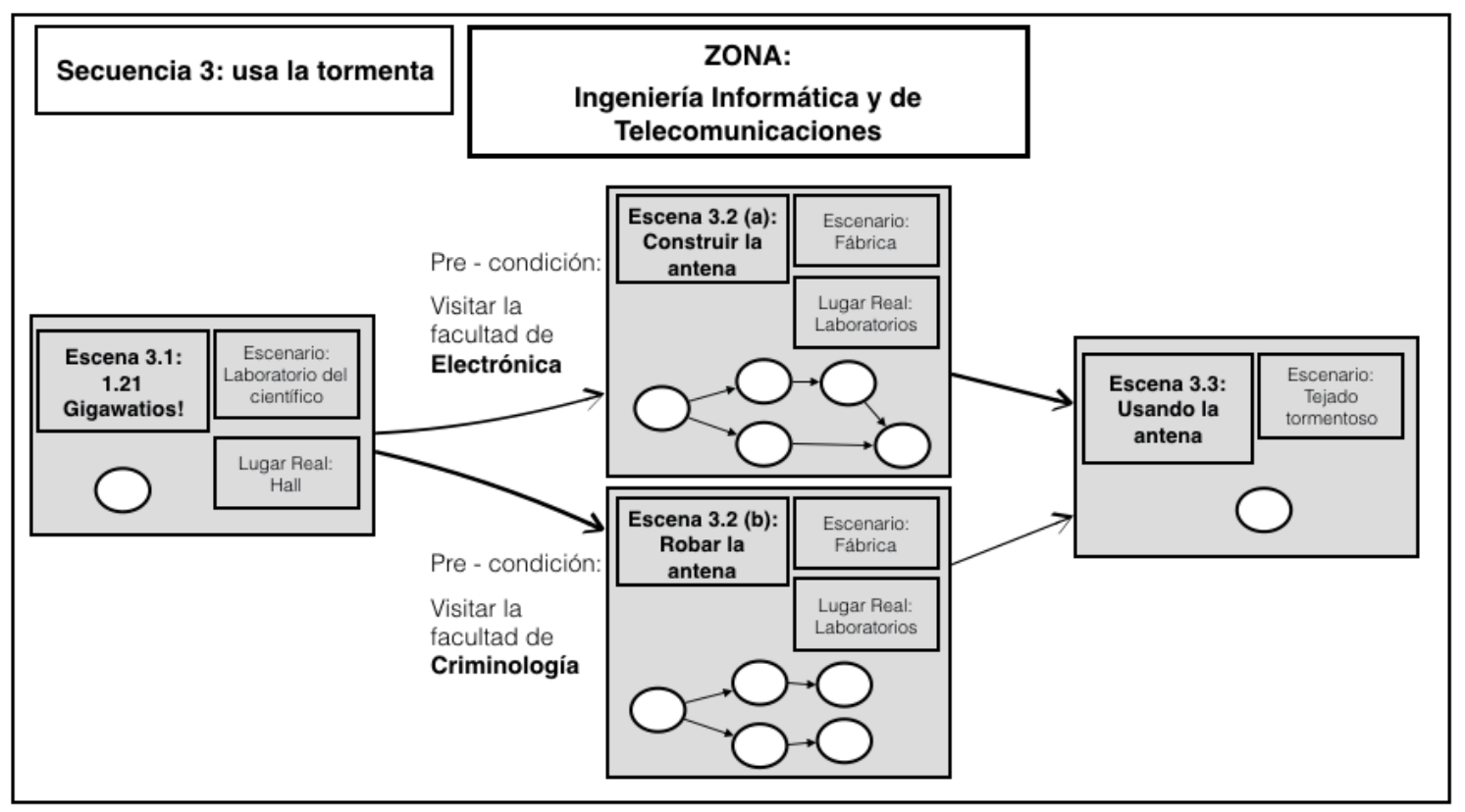

Figura 6. Ejemplo de secuencia.

Como ejemplo, nos centramos en la secuencia 3, que se muestra en una vista cercana en la Figura 6. Cuando el jugador completa la escena "1.21 Gigawatios!", hay varias opciones:

a. Si el jugador ha completado previamente en el juego la visita de la facultad de electrónica, puede construir la antena a través de varias tareas en la escena 3.2 (a).

b. Si el jugador ha completado la visita al Departamento de Criminología, puede robar la antena en la escena $3.2(\mathrm{~b})$.

c. Si el jugador ha visitado ambas, puede elegir qué camino tomar.

d. Si el jugador no ha visitado ni la Facultad de Electrónica ni el Departamento de Criminología, la información necesaria se da dentro de la escena que escoja, por lo que hay dos ramificaciones en el grafo de eventos (uno con eventos dando esa información y otro con eventos con el mismo contenido narrativo, pero sin esa información).

La opción d podría haberse solucionado forzando al jugador a visitar los lugares omitidos. Sin embargo, en esta experiencia, los lugares están muy lejos unos de otros, así que dar la información en la historia es la mejor solución.

Otro ejemplo: en la secuencia 4 (ver Figura 5), tras recoger las partes del monstruo, el jugador puede darles vida en la escena "Reanimación extraña" solo si posee el químico obtenido en la Facultad de Química. Como esta escena no está ligada a ningún lugar real, el jugador puede visitar la Facultad de Química tras la escena "Partes del cuerpo" y jugar el resto de la secuencia donde quiera que esté.

Por último, la secuencia 6 no puede ser jugada hasta que las pre-condiciones se hayan alcanzado: obtener la antena, obtener las partes del cuerpo y hacerlas funcionales y obtener el corazón.

Evaluación de la narrativa: la historia se representa mediante un grafo. En este, podemos encontrar diferentes caminos que el jugador puede tomar. Cada una de estas instancias de la historia (o historias personalizadas) puede ser evaluada de acuerdo a su intensidad emocional o a su estructura narrativa, como puede verse en [11].

Localización de la narrativa en el mundo real: en este caso, la narrativa está fuertemente ligada a lugares reales, ya que el jugador tiene que estar físicamente allí para recibir la historia. Se usarán varios dispositivos para alcanzar dicho objetivo: localización mediante GPS para las primeras escenas de las secuencias 2, 3 y 4; varios códigos QR en las escenas "Construir la antena", "Robar la antena" y en las esculturas de la escena "Partes del cuerpo", así como en la escena de la secuencia 5 "Obtener el corazón"; y sensores de proximidad en un desfibrilador real para la escena "Encontrar el desfibrilador". 
Las pre-condiciones y post-condiciones también están basadas, en su mayoría, en acciones sobre el mundo real, de modo que la evolución narrativa depende de acciones llevadas a cabo por el jugador en la realidad. Como se ha mencionado, en la secuencia 3, el uso del concepto de escena gemela se aplica a acciones del jugador sobre el mundo real. Esto se ve plasmado en las escenas 3.2 (a) y 3.2 (b). En ellas se narra la obtención de la antena de dos formas diferentes, dependiendo de los lugares reales que haya visitado previamente el jugador.

Soporte de experiencias reales: visitar las diferentes facultades, laboratorios, edificios, etc. tiene el objetivo de proporcionar al jugador conocimiento sobre ellos. En esos lugares, se interacciona con personal y estudiantes, lo cual permite enriquecer la experiencia con contenido real. Esto será parte de la historia vivida por el jugador. Además, algunos eventos físicos pueden generar evoluciones narrativas, como encontrar códigos QR o visitar lugares, gracias a la fuerte estructuración de la narrativa y al conjunto de pre y post condiciones integradas en ella.

\section{Conclusiones}

En este trabajo, hemos revisado las características principales de la narrativa geolocalizada y, en particular, cómo se utiliza en juegos pervasivos. Para ello se han revisado algunos de los trabajos más significativos en este campo. En este artículo, proponemos un modelo que permite estructurar y diseñar la narrativa interactiva sobre la que se construye la experiencia de un juego pervasivo geolocalizado. El modelo propuesto tiene las siguientes características: no linealidad, jerarquía, control de la narrativa interactiva, evaluación de la narrativa, localización de la narrativa en el mundo real y soporte de experiencias reales. Para ilustrar el modelo, se ha presentado el diseño de una historia basada en el juego presentado en [12] que implementa las características de nuestro modelo.

Nuestro trabajo para un futuro inmediato consistirá en realizar la implementación de la historia diseñada. Con ello, podremos realizar una experiencia que nos permita probar la efectividad del juego y la historia y comprobar, también, posibles errores del modelo, así como cuestionar su utilidad.

\section{Agradecimientos}

Este trabajo fue sostenido por el proyecto DISPERSA (TIN2015-67149-C3-3-R), financiado por el Plan Nacional Español de I+D+i, y el proyecto VIDEOJUEGOS-TIC (P11- TIC-7486), financiado por la Junta de Andalucía y fondos FEDER.

\section{Referencias}

[1] Markus Montola, Exploring the Edge of the Magic Circle: Defining Pervasive Games, Proceedings of Digital Arts and Culture. Copenhagen, 2005.

[2] Christian Kittl, Otto Petrovic, "Pervasive games for education", Proceedings of the 2008 Euro American Conference on Telematics and Information Systems, 2008

[3] Jesse Schell, The Art of Game Design: A Book of Lenses, by Morgan Kaufmann Publishers, 2008

[4] J.L. González, F.L. Gutiérrez, F. Montero N. Padilla, "Playability: Analyzing User Experience in Video Games", Behaviour \& Information Technology (B\&IT), Taylor \& Francis, V31(10), pp 1033-1054. (2012)

[5] Monchán, Josué (2015). "Emergencia en el apocalipsis zombi. Mecanismos para la creación de narrativas emergentes en DayZ". En: adComunica 9, págs. 185-192. 
[6] Jensen, D., Nielsen, G., \& Vutborg, R. Location-based Storytelling in the Urban Environment.

[7] Dow, S., Lee, J., Oezbek, C., Maclntyre, B., Bolter, J. D., \& Gandy, M. (2005, June). Exploring spatial narratives and mixed reality experiences in Oakland Cemetery. In Proceedings of the 2005 ACM SIGCHI.

[8] Nisi, V., Oakley, I., \& Haahr, M. (2008). Location-aware multimedia stories: turning spaces into places. Universidade Cátolica Portuguesa, 72-93.

[9] Shin, J., Kim, J., Woo. W. (2016). Narrative Design for Rediscovering Daereungwon: a location based augmented reality game.

[10] Naliuka, K., Carrigy, T., Paterson, N., \& Haahr, M. (2010, December). A narrative architecture for story-driven location-based mobile games. In International Conference on Web-Based Learning (pp. 11-20). Springer Berlin Heidelberg.

[11] López-Arcos, José Rafael; Gutiérrez Vela, Francisco Luis; Padilla-Zea, Natalia; Paderewski, Patricia (2014) A Method to Analyze Efficiency of the Story as a Motivational Element in Video Games. Proceedings of the European Conference on Games Based Learning, Vol. 2, p 705.

[12] López-Arcos, José Rafael; Gutiérrez Vela, Francisco Luis; Padilla-Zea, Natalia; Paderewski, Patricia, "Diseño de una narrativa interactiva para experiencias geolocalizadas", In Proc. Congreso Internacional de Interacción Persona-Ordenador' 16 (2016).

\section{Sobre los autores}

\section{José Rafael López Arcos}

Licenciado en Ingeniería Informática y máster en Desarrollo de software de la Universidad de Granada. Desde 2012, desarrolla su trabajo de investigación en la Universidad de Granada. Actualmente, es estudiante de doctorado y su investigación está relacionada con el diseño de contenidos narrativos interactivos y el desarrollo de videojuegos educativos.

\section{Francisco Luis Gutiérrez Vela}

Doctor en Ingeniería del software y profesor en el Departamento de Ingeniería del software y director del Grupo de Investigación en Especificación, Desarrollo y Evolución de Software (GEDES), Universidad de Granada, España. Está especializado en sistemas interactivos, interacción persona-ordenador, e-learning, aprendizaje basado en juegos y gamificación. Actualmente, está involucrado en varios proyectos de investigación (nacionales y locales) y ha escrito más de 60 artículos de investigación publicados en revistas nacionales e internacionales y conferencias especializadas en esos temas. Actualmente, está interesado en el uso de videojuegos y sistemas interactivos aplicados a la educación.

\section{Natalia Padilla-Zea}

Profesora adjunta en la Universidad Internacional de la Rioja, doctora por la Universidad de Granada e investigadora en el Instituto de Investigación, Innovación y Tecnología Educativas (iTED) del vicerrectorado de transferencia y tecnología de UNIR. Su línea de investigación se centra en el uso de videojuegos educativos, el aprendizaje colaborativo mediado por computador y metodologías de diseño de videojuegos educativos. Actualmente, imparte docencia en el grado de Ingeniería informática, así como distintos máster de la escuela superior de Ingeniería y Tecnología de UNIR.

\section{Patricia Paderewski}

(PhD., 2003) es profesora a tiempo completo en la Universidad de Granada (España). Está involucrada actualmente en varios proyectos de investigación nacionales y locales. Sus campos de investigación son la arquitectura del software, los modelos de sistemas software evolutivos, el desarrollo de aplicaciones colaborativas, e-learning y videojuegos educativos, en los cuales ha escrito más de 80 artículos de investigación, publicados en revistas nacionales e internacionales y conferencias especializadas en esos temas. 


\section{Nuria Medina Medina}

Recibió su doctorado en Ciencias de la computación de la Universidad de Granada (UGR), en 2004, proponiendo un modelo adaptativo y evolutivo para sistemas hipermedia. Actualmente, pertenece al equipo de dirección del Centro de Investigación en Tecnologías de la Información y las Comunicaciones (CITIC-UGR) y es profesora en el Departamento de Lenguajes y Sistemas Informáticos en esta Universidad. Adicionalmente, es miembro del grupo de investigación GEDES, donde investiga en juegos serios y ha dirigido un proyecto que implementa juegos educativos en clases de colegios andaluces (P11TIC7486). 